\title{
$B(S, \Sigma)$ ALGEBRAS
}

K. G. JOHNSON

In the present paper the closed ideals of $B(S, \Sigma)$ type spaces [1] are considered. These ideals are characterized and a representation theorem for the quotient algebra with respect to such an ideal is obtained. The latter is useful in obtaining a representation of the dual of the quotient algebra.

\section{Closed ideals.}

(1.1) Definition. Let $S$ be an arbitrary set, and $\Sigma$ a Boolean ring of subsets of $S$. The algebra $B(S, \Sigma)$ consists of all uniform limits of finite linear combinations of characteristic functions of sets in $\Sigma$. The norm in $B(S, \Sigma)$ is given by the formula $\|f\|=\operatorname{Sup}_{s \in S}|f(s)|$.

This definition is slightly more general than that given in [1] where $\Sigma$ is required to be a field of sets (Boolean ring with identity = Boolean algebra).

(1.2) Definition. A simple function in $B(S, \Sigma)$ is a finite linear combination of characteristic functions of sets in $\Sigma$.

(1.3) Lemma. $B(S, \Sigma)$ has an identity if and only if $\Sigma$ has an identity.

Proof. Obvious.

(1.4) THEOREM. There is a norm preserving isomorphism between $B(S, \Sigma)$ and the algebra $C(X)$ of functions which vanish at infinity on a totally disconnected locally compact hausdorff space $X . X$ has a basis of both open and closed sets which is isomorphic to $\Sigma$. If $B(S, \Sigma)$ is complex this algebra $C(X)$ is the complex algebra. If $B(S, \Sigma)$ is real $C(X)$ is the real algebra.

Proof. $B(S, \Sigma)$ satisfies conditions which are known from the theory of Gel'fand representation [2] or [3] to insure that $B(S, \Sigma)$ is isometrically isomorphic to a $C(X)$ where $X$ is locally compact. We need but show that $X$ is totally disconnected. If $f \in B(S, \Sigma)$ let $\hat{f}$ be its image in $C(X)$. If $f$ is a characteristic function $f^{2}=f$ implies $\hat{f}^{2}=\hat{f}$. Therefore $\hat{f}$ is a characteristic function. Since $\hat{f}$ is continuous it must be the characteristic function of a both open and closed set.

This generalizes immediately to the conclusion that if $f$ is a simple function then so is $\hat{f}$. Let $U$ be an open set in $X$ and let $x \in U$. There exists an open $V$ such that closure $(V)$ is compact, $x \in V$ and $V \subset U$. By Urysohn's Lemma there is a continuous function $\hat{g}$ in $C(X)$ such

Received by the editors January 18, 1963. 
that $\hat{g}(x)=1, \hat{g}=0$ on $X-V$. Since the corresponding $g$ in $B(S, \Sigma)$ can be approximated uniformly by simple functions $g_{n}$, it follows that their corresponding $\hat{g}_{n}$ can be used to approximate $\hat{g}$ uniformly. Thus there exists a simple $\hat{g}_{n}$ such that $\left\|\hat{g}-\hat{g}_{n}\right\|<\frac{1}{2} . \hat{g}_{n}=\sum_{i=1}^{i=m} a_{i} \chi_{S_{i}}$, where the $S_{i}$ are pairwise disjoint and each is both open and closed. It is clear that $x$ is in one of the $S_{i}$, say $S_{t}$, and $S_{t} \subset V \subset U$. Since $S_{t}$ is both open and closed we have proved the desired total disconnectedness.

The next result is true of any ideal $I$ in $B(S, \Sigma)$ whether $I$ is closed or not.

(1.5) Lemma. Let $I$ be an ideal of $B(S, \Sigma)$. Then there is an ideal $\Sigma^{\prime}$ of $\Sigma$ such that I contains all finite linear combinations of characteristic functions of sets in $\Sigma^{\prime}$ and $I \subset B\left(S, \Sigma^{\prime}\right)$.

Proof. It will suffice to prove this for the $C(X)$ of (1.4). Let $f \in I$ and let $\epsilon$ be any positive number. There is a simple $f_{\epsilon}$ in $C(X)$ such that $\left\|f-f_{\epsilon}\right\|<\epsilon$ and $f_{\epsilon}(x) \neq 0$ implies $f(x) \neq 0$. It can be assumed that the sets whose characteristic functions are involved in $f_{\mathrm{e}}$ are disjoint. Let $S$ be one of them. Then $\chi_{s}$ is continuous. Since $f(s) \neq 0$ for $s \in S$ it follows that $1 / f \cdot \chi_{S}$ is continuous if $1 / f \cdot \chi_{S}(x)$ is defined equal to zero whenever $x \in S$. It follows that $f \cdot 1 / f \cdot \chi_{S}=\chi_{s} \in I$. This proves that any $f \in I$ can be uniformly approximated by finite linear combinations of characteristic functions in $I$. It but remains to verify that the collection of sets underlying the characteristic functions is an ideal in $\Sigma$. This verification being easy and straightforward is omitted.

The next result characterizes the closed ideals in $B(S, \Sigma)$ and shows that the structure of the lattice (with respect to set theoretic inclusion) of closed ideals in $B(S, \Sigma)$ is the same as that of the lattice of ideals in $\Sigma$. Each ideal in $\Sigma$ is identified with a closed ideal of $B(S, \Sigma)$ and conversely each closed ideal in $(B, S, \Sigma)$ is determined by one ideal in $\Sigma$.

(1.6) Theorem. Let $\Sigma^{\prime}$ be an ideal in $\Sigma$. Then (1) $B\left(S, \Sigma^{\prime}\right)$ is a closed ideal in $B(S, \Sigma)$; (2) If $\Sigma^{\prime}$ and $\Sigma^{\prime \prime}$ are distinct ideals in $\Sigma$ then $B\left(S, \Sigma^{\prime}\right)$ and $B\left(S, \Sigma^{\prime \prime}\right)$ are distinct; (3) If $I$ is a closed ideal in $B(S, \Sigma)$ then there is an ideal $\Sigma^{\prime \prime \prime}$ of $\Sigma$ such that $I=B\left(S, \Sigma^{\prime \prime \prime}\right)$.

Proof. Since both $B(S, \Sigma)$ and $B\left(S, \Sigma^{\prime}\right)$ have dense collections of simple functions to prove (1) it suffices to show that if $f$ and $f^{\prime}$ respectively are simple functions in $B(S, \Sigma)$ and $B\left(S, \Sigma^{\prime}\right)$ respectively then $f \cdot f^{\prime} \in B\left(S, \Sigma^{\prime}\right)$. This, however, follows from the fact that $\Sigma^{\prime}$ is an ideal in $\Sigma$. (2) If $\Sigma^{\prime}$ and $\Sigma^{\prime \prime}$ are distinct then there is an $S$ in one not in the 
other. Suppose $S \in \Sigma^{\prime}, S \in \Sigma^{\prime \prime}$. Then $\chi_{S} \in B\left(S, \Sigma^{\prime}\right), \chi_{s} \notin B\left(S, \Sigma^{\prime \prime}\right)$. Hence $B\left(S, \Sigma^{\prime}\right) \neq B\left(S, \Sigma^{\prime \prime}\right)$. (3) By (1.5) there is an ideal $\Sigma^{\prime \prime \prime}$ of $\Sigma$ such that $I$ contains all finite linear combinations of characteristic functions in $\Sigma^{\prime \prime \prime}$ and $I \subseteq B\left(S, \Sigma^{\prime \prime \prime}\right)$. If $I$ is closed it follows that $I=B\left(S, \Sigma^{\prime \prime \prime}\right)$.

(1.7) Corollary. Let $\Sigma^{\prime}$ be an ideal in $\Sigma$. Then each closed ideal in $B\left(S, \Sigma^{\prime}\right)$ is a closed ideal in $B(S, \Sigma)$.

Proof. If $I$ is a closed ideal in $B\left(S, \Sigma^{\prime}\right)$ then by part (3) of (1.6) there is a $\Sigma^{\prime \prime}$ which is an ideal of $\Sigma^{\prime}$ such that $I=B\left(S, \Sigma^{\prime \prime}\right)$. But $\Sigma^{\prime \prime}$ is also an ideal of $\Sigma$. Hence $B\left(S, \Sigma^{\prime \prime}\right)$ is an ideal in $B(S, \Sigma)$ by part (1) of (1.6).

(1.8) Corollary. $\Sigma^{\prime}$ is a maximal ideal in $\Sigma$ if and only if $B\left(S, \Sigma^{\prime}\right)$ is a maximal ideal in $B(S, \Sigma)$.

(1.9) Corollary. Let $\Sigma^{\prime}$ be a Boolean ring of subsets without identity. Then $\Sigma^{\prime}$ can be imbedded in a Boolean algebra of subsets $\Sigma$ such that $\Sigma^{\prime}$ is a maximal proper ideal of $\Sigma$ and $B\left(S, \Sigma^{\prime}\right)$ is a maximal proper ideal in $B(S, \Sigma)$.

Proof. Define $\Sigma$ by $\Sigma=\Sigma^{\prime} \cup\left\{\sigma_{i} \cup\left(S \backslash \sigma_{j}\right) \mid \sigma_{i}, \sigma_{j} \in \Sigma^{\prime}\right\}$. It is well known and in fact easily verified that $\Sigma$ is a Boolean algebra and $\Sigma^{\prime}$ is a maximal ideal in $\Sigma$. The rest of the assertion follows from (1.8).

\section{Quotient algebras.}

(2.1) Theorem. Let $I$ be a closed ideal in $B(S, \Sigma)$ and $\Sigma^{\prime}$ be the ideal of $\Sigma$ such that $I=B\left(S, \Sigma^{\prime}\right)$. Let $B(S, \Sigma) \bmod I$ be the quotient algebra of $B(S, \Sigma)$ with respect to $I$. Then there exists a set $T$ with a Boolean ring $\Pi$ of subsets of $T$ such that $B(S, \Sigma) \bmod I$ is isomorphic to $B(T, \Pi)$ and $\Pi$ is isomorphic to $\Sigma \bmod \Sigma^{\prime}$ and such that the isomorphism between $B(S, \Sigma) \bmod I$ and $B(T, \Pi)$ is an isometry.

Proof. By Stone's theorem, $\Sigma$ mod $\Sigma^{\prime}$ is isomorphic to a Boolean Ring $\Pi$ of subsets of a set $T$. The elements of $B(S, \Sigma)$ mod $I$ are cosets. Call an element of $B(S, \Sigma) \bmod I$ a simple element if it contains a simple function of $B(S, \Sigma)$. It is clear that the simple elements in $B(S, \Sigma) \bmod I$ are dense. It will suffice to show that the ring of simple elements in $B(S, \Sigma)$ mod $I$ is isomorphic to the ring of simple functions in $B(T, \Pi)$ and that the isomorphism say $F$ is an isometry. Let $x$ be a nonzero simple element in $B(S, \Sigma) \bmod I$. Now $x$ contains a simple function $f$ where $f=\sum_{i=1}^{i=r} a_{i} \chi_{S_{i}}+\sum_{i=r+1}^{i=n} a_{i} \chi_{S_{i}}$, where $S_{i} \in \Sigma$, each $i, S_{i} \notin \Sigma^{\prime}$ if $i \leqq r$, whereas $S_{i} \in \Sigma^{\prime}$ if $i>r$. It is not hard to see that $\|x\|=\left\|\sum_{i=1}^{i=r} a_{i} \chi_{s_{i}}\right\|$ in $B(S, \Sigma)$. This follows from the fact that 


$$
\|x\|=\begin{aligned}
& \inf \left\|f+g_{n}\right\| \\
& \text { simple } g_{n} \in I .
\end{aligned}
$$

(The density of the simple $g_{n}$ in $I$ due to (1.5) implies no loss in the assumption that the $g_{n}$ are simple in the expression.)

Let $T_{i} \in \Pi$ be the image of $S_{i} \bmod \Sigma^{\prime}$ by the Stone representation. Define $F(x)=\sum_{i=1}^{i=r} a_{i} \chi_{T_{i}}$. Simple verification shows that if $f_{1}$ and $f_{2}$ are both simple and belong to $x$ then both yield the same $F(x)$. Hence $F$ is a function. It is clearly onto. The remaining details proving that it is an isomorphism are easy and are omitted. The isometry follows from $\|x\|=\left\|\sum_{i=1}^{i=r} a_{i} \chi_{S_{i}}\right\|=\left\|\sum_{i=1}^{i=r} a_{i} \chi_{t i}\right\|$, where the first equality was shown above and the latter is easily verified.

(2.2) Theorem. Let $I$ be a closed ideal in $B(S, \Sigma)$ and $\Sigma^{\prime}$ the ideal of $\Sigma$ such that $I=B\left(S, \Sigma^{\prime}\right)$. Let $B(S, \Sigma) \bmod I$ be the quotient algebra of $B(S, \Sigma)$ with respect to $I$. Then $B(S, \Sigma) \bmod I$ is isomorphic to a $C(X)$ where $X$ is totally disconnected and has a basis of both open and closed sets isomorphic to $\Sigma \bmod \Sigma^{\prime}$. The isomorphism is an isometry.

Proof. By (2.1) it suffices to observe that $B(T, \Pi)$ is isomorphic to a $C(X)$ and a basis of both open and closed sets in $X$ is isomorphic to II. This follows from (1.4).

Dunford and Schwartz [1] discuss a representation of $B^{*}(S, \Sigma)$, the dual space of $B(S, \Sigma)$, due to Hildebrandt [4] and Fichtenholz and Kantorovich [5]. The discussion in [1] presumably is concerned only with $B(S, \Sigma)$ with identity; however the discussion there of $B^{*}(S, \Sigma)$ is general, in fact the only properties of $\Sigma$ used in its discussion is the fact that $\Sigma$ is a Boolean ring. Consequently the following can be stated and the reader can be referred to $[1$, p. 258] for the proof.

(2.3) Theorem. There is an isometric isomorphism between $B^{*}(S, \Sigma)$ and $b a(S, \Sigma)$, the space of bounded additive set function on $\Sigma$, determined by the identity (D) $L^{*} f=\int_{S} f(s) \mu(d s)$. Thus for each $L^{*}$ in $B^{*}(S, \Sigma)$ there is a unique $\mu$ in $b a(S, \Sigma)$ such that (D) holds; for each $\mu$ in ba $(S, \Sigma)$ there is a unique $L^{*}$ such that (D) holds; and the correspondence between $L^{*}$ and $\mu$ is linear and isometric.

(2.4) Definition. Let $\Sigma^{\prime}$ be an ideal of a Boolean ring of subsets $\Sigma$ of a set $S$. Then $b a\left(S, \Sigma \bmod \Sigma^{\prime}\right)$ is the collection of bounded additive set functions on $\Sigma$ which vanish on sets in $\Sigma^{\prime}$.

(2.5) Theorem. Let $\mathfrak{L}^{*}$ designate the space of bounded linear functionals on the quotient algebra $B(S, \Sigma) \bmod B\left(S, \Sigma^{\prime}\right)$.

Then there is an isometric isomorphism between $\mathcal{L}^{*}$ and ba $\left(S, \Sigma \bmod \Sigma^{\prime}\right)$ determined by the identity (D) $L^{*}[f]=\int_{S} f(s) \mu(d s)$, where $f$ is any element in $[f]$. For each $L^{*}$ in $\mathscr{L}^{*}$ there is a unique $\mu$ in $b a\left(S, \Sigma \bmod \Sigma^{\prime}\right)$ 
such that $(\mathrm{D})^{\prime}$ holds; for each $\mu$ in $b a\left(S, \Sigma \bmod \Sigma^{\prime}\right)$ there is a unique $L^{*}$ such that (D)' holds; and the correspondence between $L^{*}$ and $\mu$ is linear and isometric.

Proof. Represent $B(S, \Sigma) \bmod B\left(S, \Sigma^{\prime}\right)$ as a $B(T$, II) by using (2.1). Using the fact that $\Pi$ is isomorphic to $\Sigma \bmod \Sigma^{\prime}$ the result desired follows from an application of $(2.3)$ to $B(T, \Pi)$.

Let $I$ be a bounded closed interval in $E^{m}$. The author has shown in [6] that the algebra of bounded real-valued Riemann integrable functions $R(I)$ consists of all uniform limits of real finite linear combinations of characteristic functions of sets in $I$ having Jordan content. Designating the collection of sets having Jordan content by $J$ we have $R(I)=B(I, J)$ in the notation of the present paper. Define $R_{\infty}(I)$ to be the collection of classes in $R(I)$ such that $f_{1}$ and $f_{2}$ are in the same class if and only if $f_{1}-f_{2}$ vanishes everywhere except on a set of Lebesgue measure zero. Let $N$ designate the collection of sets in $I$ of Lebesgue measure zero. Then $J \cap N$ in the ring of sets of Jordan content zero. Let $R_{\infty}(I)$ have the norm defined by $\|[f]\|=\inf S \in N \sup X$ $\notin S|f(X)|=\|f\|_{\infty}$, where $f$ is any function in $[f]$. It is shown in [6] that $B(I, J \cap N)$ is a closed ideal in $B(I, J)$ and that with the norm just given above $R_{\infty}(I)=B(I, J) \bmod B(I, J \cap I)=R(I) \bmod B(I, J \cap I)$. The following result announced in [6] then follows from (2.6).

(2.6) Theorem. Let $R_{\infty}^{*}(I)$ designate the dual space of $R_{\infty}(I)$. Then there is an isometric isomorphism between $R_{\infty}^{*}(I)$ and (real) $b a(I, J \bmod J \cap N)$ determined by the identity $(\mathrm{D})^{\prime} L^{*}[f]=\int_{I} f(x) \mu(d x)$ when $f$ is any element in $[f]$. Thus for each $L^{*}$ in $R_{\infty}^{*}(I)$ there is a unique $\mu$ in $b a(I, J \bmod J \cap N)$ such that (D)' holds; for each $\mu$ in $b a(I, J \bmod J \cap N)$ there is a unique $L^{*}$ such that $(\mathrm{D})^{\prime}$ holds; and the correspondence between $L^{*}$ and $\mu$ is linear and isometric.

\section{REFERENCES}

1. N. Dunford and Jacob T. Schwartz, Linear operators. I, Interscience, New York, 1958.

2. Charles E. Rickart, General theory of Banach algebras, Van Nostrand, New York, 1960.

3. L. H. Loomis, An introduction to abstract harmonic analysis, Van Nostrand, New York, 1953.

4. T. H. Hildebrandt, On bounded functional operations, Trans. Amer. Math. Soc. 36 (1934), 868-875.

5. G. Fichtenholz and L. V. Kantorovitch, Sur les opérations linéaires dans l'espace des fonctions bornées, Studia Math. 5 (1934), 69-98.

6. K. G. Johnson, Algebras of Riemann integrable functions, Proc. Amer. Math. Soc. 13 (1962), 437-441.

\section{Miami University}

